\title{
Clinical and magnetic resonance imaging features of 14 patients with trilateral retinoblastoma
}

\author{
Ting Gui ${ }^{1} \wedge$, Hui Zheng ${ }^{1}$, Ming Liu ${ }^{1}$, Zhengrong Xia ${ }^{1}$, Xunda $\mathrm{Ji}^{2}$, Qiufeng Yin ${ }^{1}$, Dengbin Wang ${ }^{1}$, \\ Yuhua $\mathrm{Li}^{1}$, Shuxian Chen ${ }^{3}$ \\ ${ }^{1}$ Department of Radiology, Xinhua Hospital Affiliated to Shanghai Jiaotong University School of Medicine, Shanghai, China; ${ }^{2}$ Department of \\ Ophthalmology, Xinhua Hospital Affiliated to Shanghai Jiaotong University School of Medicine, Shanghai, China; ${ }^{3}$ Department of Oncology, \\ Xinhua Hospital Affiliated to Shanghai Jiaotong University School of Medicine, Shanghai, China
}

Correspondence to: Shuxian Chen. Department of Oncology, Xinhua Hospital Affiliated to Shanghai Jiaotong University School of Medicine, Shanghai 200092, China. Email: chenshuxian@xinhuamed.com.cn; Yuhua Li. Department of Radiology, Xinhua Hospital Affiliated to Shanghai Jiaotong University School of Medicine, Shanghai 200092, China. Email: liyuhua@xinhuamed.com.cn.

Background: Our study aimed to comprehensively investigate the age of onset, magnetic resonance imaging (MRI) features, and prognosis of children with trilateral retinoblastoma (TRB).

Methods: We included 14 patients with TRB diagnosed or followed up in our hospital. The age of onset and MRI features of the intraocular tumor and intracranial lesions were evaluated. A follow-up study was also conducted.

Results: A total of 11 participants were diagnosed with concurrent TRB at the age of $11.1 \pm 7.4$ months, and 3 participants had late-onset TRB at age $37 \pm 19.1$ months. The incidence of TRB with unilateral eye involvement was $7.1 \%$ (1/14). The intraocular tumors showed intense enhancement in contrast-enhanced T1-weighted images (WI) and significant diffusion restriction in diffusion WI (DWI) with an apparent diffusion coefficient (ADC) of $(0.619 \pm 0.22) \times 10^{-3} \mathrm{~mm}^{2} / \mathrm{s}$. The intracranial lesions showed similar DWI aspects with an ADC value of $(0.680 \pm 0.206) \times 10^{-3} \mathrm{~mm}^{2} / \mathrm{s}$. Therapeutically, 8 participants had a period of intraocular tumor stabilization and significant intracranial lesion volume reduction after chemotherapy, and 6 participants had given up treatment. Only 2 participants who simultaneously received high-dose chemotherapy and autologous hematopoietic stem cell rescue were still alive with no recurrence at 24 and 54 months of follow-up. The 1-, 2-, and 3-year overall survival (OS) rates were $80 \%, 18.75 \%$, and $12.5 \%$, respectively.

Conclusions: Patients with unilateral or bilateral RB can develop TRB. The intraocular and intracranial tumors showed slightly different ADC values. High-dose chemotherapy, combined with stem cell rescue can significantly improve survival. A long term and scheduled follow-up before 60 months of age is necessary for screening later-onset TRB patients.

Keywords: Retinoblastoma; trilateral retinoblastoma (TRB); quadrilateral retinoblastoma; magnetic resonance imaging (MRI); children

Submitted Apr 22, 2020. Accepted for publication Oct 27, 2020.

doi: 10.21037/qims-20-605

View this article at: http://dx.doi.org/10.21037/qims-20-605

\footnotetext{
^ ORCID: 0000-0002-6845-7299.
} 


\section{Introduction}

Retinoblastoma is the most common intraocular childhood malignancy, with a prevalence of 1 in 18,000 children aged $<5$ years (1-5). It is initiated by a mutation of the retinoblastoma 1 (RB1) gene, which was the first described tumor-suppressor gene (2). Trilateral retinoblastoma (TRB) has been estimated to occur in $0.5-6 \%$ of patients with bilateral retinoblastoma (6-8); TRB refers to the development of a primary intracranial primitive neuroectodermal tumor in a patient with intraocular RB and was first described in 1971 by Jacobiec et al. (1,7,9-14). With rare exceptions, TRB is located in the pineal gland or the suprasellar or parasellar region, and the presence of a second midline tumor in the suprasellar or parasellar region is referred to as quadrilateral retinoblastoma (QRB). An associated midline intracranial tumor is not a metastasis, and it represents multifocal disease. However, the origin of intracranial tumors in RB patients is still uncertain (15). It has been hypothesized that intracranial tumors originate from ectopic foci of retinal cells in the third ventricle floor or from pineal photoreceptors, which are functionally and morphologically similar to retinal photoreceptors. The retina and pineal gland's common photoreceptor origin may account for this susceptibility to coexistent disease (16).

The reported survival rate for patients with unilateral $\mathrm{RB}$ is approximately $93 \%$ at 5 years, and that for bilateral $\mathrm{RB}$ is approximately $92 \%$ at 5 years (15). TRB's prognosis is much poorer, especially when the tumor spreads to the subarachnoid space $(9,15)$. Yamanaka et al. conducted a systematic review of 72 TRB studies and found that the median survival for patients with a pineal tumor was 9.4 months, with a 5 -year survival rate of $15.5 \%$. The median survival was 12 months for patients with sellar tumors, and the 5 -year survival rate was $17.8 \%(1,17)$. However, we have that TRB studies have mostly been based on case reports, and only a small number of articles have analyzed the survival or treatment of TRB patients as a single independent study.

This study retrospectively analyzed the clinical and imaging features of 14 participants with T/Q RB diagnosed in Xinhua Hospital Affiliated to Shanghai Jiaotong University School of Medicine from January 2012 to December 2019, aiming to understand the clinical features and magnetic resonance imaging (MRI) findings better and to improve the survival of TRB patients.

\section{Methods}

\section{Participant population}

We included 14 patients with T/Q RB among 1,392 patients with $\mathrm{RB}$, who were initially diagnosed or underwent longterm follow-up at our hospital between January 2012 and December 2019. All of the participants' parents had denied any history of RB. The Ethics Committee approved this study of Xinhua Hospital Affiliated to Shanghai Jiaotong University School of Medicine (XHEC-D-2020-124) and was conducted following the Declaration of Helsinki and International Ethical Guidelines for Health-related Research Involving Humans. Informed consent was provided by each subject or their responsible guardian.

\section{Imaging protocols}

The MRI was performed under sedation using a 3.0-T MR scanner (Signa HDx; GE Medical Systems, Milwaukee, WI, USA) with an 8-channel head coil. Axial unenhanced T1-weighted spin-echo images with a repetition time (TR)/echo time (TE) of 400-560/9-14 ms and T2-weighted fast spin-echo images (TR/TE of 3,000-3,700/80-110) with fat saturation were obtained. Images were obtained in $\geq 2$ planes with a $3-\mathrm{mm}$ slice thickness and a $0.5-\mathrm{mm}$ interslice gap.

Diffusion-weighted imaging (DWI) images were acquired in the axial plane before administration of contrast medium using a single-shot echo-planar imaging sequence [TR/TE effective range of 3,200-5,000/70-100 ms; slice thickness of $3 \mathrm{~mm}$; a gap of $0.5 \mathrm{~mm}$; the field of view (FOV), $18-24 \mathrm{~cm}$; matrix, $128 \times 128$ ]. The b-values corresponding to the diffusion-sensitizing gradient were 0 and $1,000 \mathrm{~s} / \mathrm{mm}^{2}$ and was applied in 3 orthogonal directions.

After intravenous administration of $0.1 \mathrm{mmol} / \mathrm{kg}$ gadopentetate dimeglumine, we obtained the axial contrastenhanced T1-weighted images (T1WI) with fat saturation of all participants (TR/TE of 400-575/13-15 ms; slice thickness of $2 \mathrm{~mm}$; a gap of $0.5 \mathrm{~mm}$; the field of view, 18-24 cm; matrix, 256×256). Oblique sagittal contrastenhanced T1WI with fat saturation, parallel to the optic nerve, were also obtained using the same parameters. Axial contrast-enhanced T1-weighted images of the head without fat saturation were obtained with 5 -mm section thicknesses and $1-\mathrm{mm}$ intersection gaps. 
Table 1 Patient characteristics and outcomes

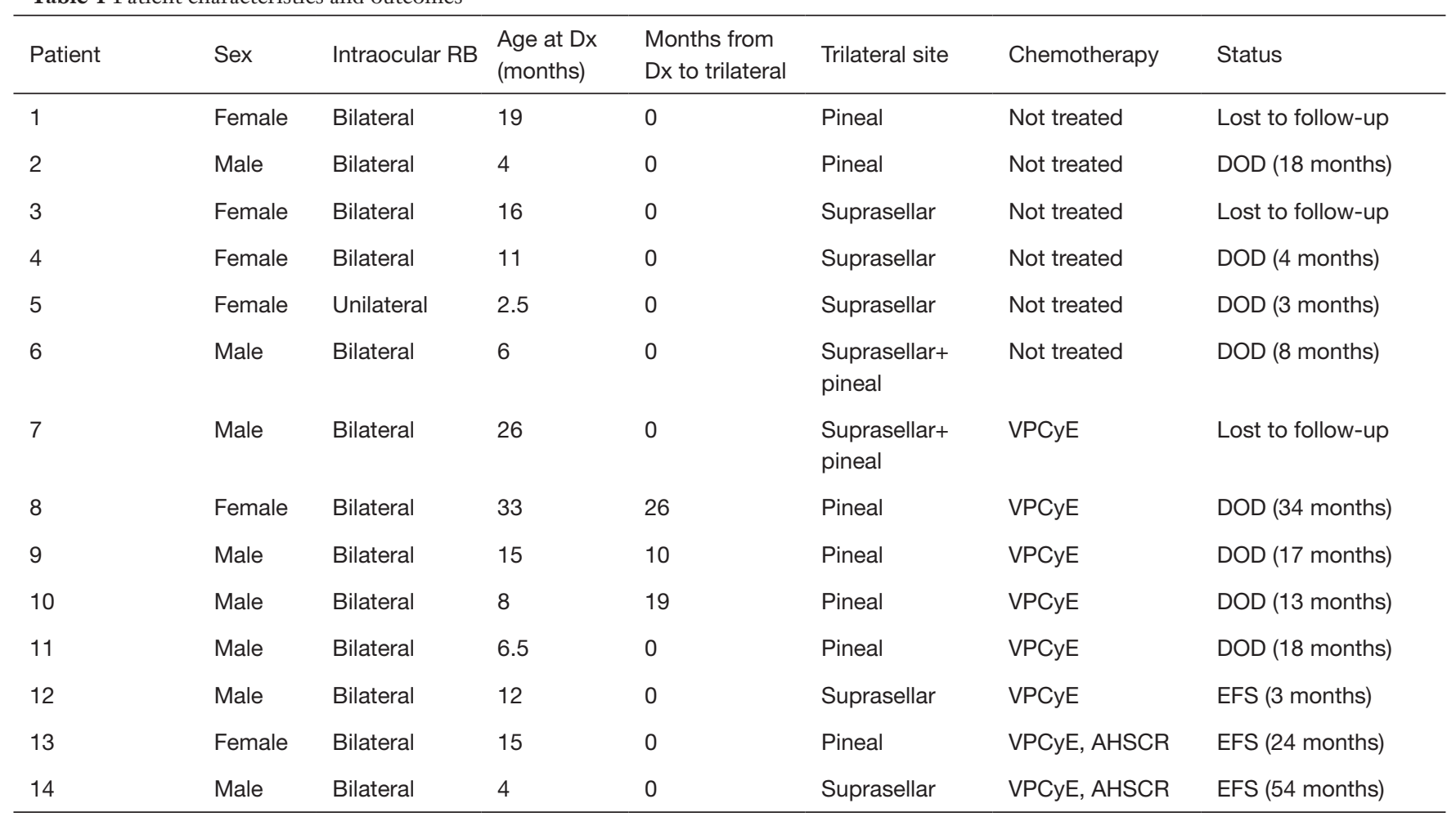

RB, retinoblastoma; Dx, diagnosis; V, vincristine; P, cisplatin; Cy, cyclophosphamide; E, etoposide; EFS, event-free survival; DOD, died of disease; AHSCR, high-dose chemotherapy with autologous hematopoietic stem cell rescue.

Table 2 Clinical features of trilateral/quadrilateral retinoblastoma

\begin{tabular}{ll}
\hline Feature & Outcome \\
\hline Patients (n) & 14 \\
Incidence in RB & $1 \%$ \\
Incidence in bilateral RB & $3.5 \%$ \\
TRB (n) & 12 \\
QRB (n) & 2 \\
Age at Dx of intraocular RB (months) & $12.7 \pm 8.8$ \\
Age at Dx of TRB/QRB (months) & $16.6 \pm 14.8$ \\
Months from Dx to TRB/QRB (months) & $18.3 \pm 8.0$ \\
Age of concurrent TRB/QRB (months) & $11.1 \pm 7.4$ \\
Age of later onset TRB (months) & $37 \pm 19.1$ \\
1-year OS rate & $80.00 \%$ \\
2-year OS rate & $18.75 \%$ \\
3-year OS rate & $12.50 \%$ \\
\hline
\end{tabular}

$\mathrm{RB}$, retinoblastoma; TRB, trilateral retinoblastoma; $\mathrm{QRB}$, quadrilateral retinoblastoma; Dx, diagnosis; OS, overall survival.

\section{Treatment}

Chemotherapy was administered to 8 participants, and 6 participants had given up treatment due to treatment costs and poor prognostic expectations. Among the 8 participants who received chemotherapy, 6 received vincristine, cisplatin, cyclophosphamide, and etoposide; and 2 received highdose chemotherapy and autologous hematopoietic stem cell rescue.

\section{Results}

\section{Clinical characteristics}

The incidence of TRB and QRB in our hospital was $1 \%(14 / 1,392)$ in all RB patients and 3.5\% (14/403) in all bilateral RB patients. The clinical characteristics of the 14 participants are summarized in Tables 1,2, and Table S1. Briefly, there were 7 participants with pineal TRB (participants 1, 2, 8-11, and 13), 5 with suprasellar TRB (participants 3-5, 12, and 14), and 2 with QRB (participants 
6 and 7). The age at first diagnosis of intraocular RB was $12.7 \pm 8.8$ months. A total of 11 participants had $\mathrm{TRB} / \mathrm{QRB}$ at the initial diagnosis of intraocular RB, at the age of $16.6 \pm 14.8$ months; and 3 had a later onset at $37 \pm 19.1$ months. The time from diagnosis of intraocular disease to later-onset TRB disease was $18.3 \pm 8.0$ months. Only 2 participants with intraocular RB, at the ages of 6 and 26 months, had quadrilateral RB at initial diagnosis. The mean age at suprasellar TRB diagnosis was 9.1 \pm 5.6 months, compared with $22.2 \pm 18.4$ months for pineal TRB.

\section{MRI characteristics of intraocular lesions}

For the 11 patients who had intraocular RB associated with concurrent TRB/QRB, the affected 20 eyes (one was a unilateral lesion, and one eye was detected by ophthalmoscopy; participants 5 and 6 , respectively) were classified according to the International Classification of Retinoblastoma (ICRB) as group A $(n=1)$, group B $(n=2)$, group $C(n=2)$, group $D(n=8)$, and group $E(n=7)$. In group A (participant 6), the lesion was too small to be detected by MRI imaging and was detected only by ophthalmoscopy. The eyeball MRI in the other 4 groups showed glaucoma in 5 eyes, eyeball atrophy in 1 eye, and subretinal hemorrhage in 2 eyes. Intraocular tumors showed medium signal intensity in T1WI, heterogeneous low signal intensity in T2WI, intense enhancement in contrastenhanced T1WI, and significant diffusion restriction in DWI with an apparent diffusion coefficient (ADC) of $(0.619 \pm 0.22) \times 10^{-3} \mathrm{~mm}^{2} / \mathrm{s}$ (Table S1).

The 3 participants with later-onset pineal TRB were initially diagnosed with bilateral RB and underwent chemotherapeutic treatment. After progression-free survival (PFS) of 10-26 months, MRI images revealed that a pineal tumor had emerged, but the bilateral intraocular lesions remained stable (Figure 1).

\section{MRI characteristics of intracranial lesions}

The intracranial lesions of 7 participants were located in the pineal region (participants 1, 2, 8-11, and 13), 5 in the suprasellar area (participants $3-5,12$, and 14), and 2 in the pineal and suprasellar regions concurrently (participants 6 and 7). The MRI results of these lesions showed circumscribed round or oval masses with an average length of $17.4 \pm 10.4 \mathrm{~mm}$. The intracranial lesions demonstrated homogeneous medium signal intensity or heterogeneously mixed signal intensity in T1WI/T2WI, owing to intratumoral hemorrhage or calcification, and intense enhancement in contrast-enhanced T1WI. The lesions were also investigated with DWI, and the results showed significant diffusion restriction with an ADC of $(0.680 \pm 0.206) \times 10^{-3} \mathrm{~mm}^{2} / \mathrm{s}$ (Table S1).

Leptomeningeal and cerebrospinal dissemination was found in 1 pineal TRB and 1 suprasellar TRB participant (participants 1 and 5; Figures 2 and 3; respectively).

\section{Treatment outcome and overall survival (OS)}

Among the 14 participants, 6 had given up any treatment due to high treatment costs, coupled with poor prognostic expectations (participants 1-6). Of these 6, 2 who had given up treatment had leptomeningeal and cerebrospinal dissemination at the time of origin; 1 was lost to followup (participant 1; Figure 2), and 1 died due to disease progression after 3 months (participant 5; Figure 3). The 3 remaining participants died $4-18$ months after followup due to disease progression (participants 2, 4, and 6), including a QRB participant (participant 6; Figure 4), and 1 was lost to follow-up (participant 3).

The remaining 8 participants received active chemotherapy (participants 7-14). Vincristine, cisplatin, cyclophosphamide, and etoposide was received by 6 participants (participants 7-12), and 2 simultaneously received high-dose autologous hematopoietic stem cell rescue (participants 13 and 14). Among these 8 participants, contact with 1 case of QRB was lost during the follow-up (participant 7). In the other 5 participants (participants 8-12), including 3 cases of later-onset pineal TRB, the intracranial tumors had significantly shrunk after standardized chemotherapy, and the intraocular lesions reached a stable stage (approximately 8-19 months). However, except for 1 participant (participant 12) who had undergone treatment and was followed up for only 3 months, these 4 participants died of tumor recurrence and cerebrospinal fluid dissemination during the continuous period (3-15 months) of follow-up (Figure 5). For the 2 participants who had received autologous hematopoietic stem cell rescue in addition to high-dose chemotherapy, the intraocular and intracranial lesions had all significantly shrunk, and they were all alive without recurrence for 24 and 54 months (participant 13 and 14, respectively, Figure 6). The 1-, 2-, and 3-year OS rates were $80 \%$, $18.75 \%$, and $12.5 \%$ in our study. There were no participant 

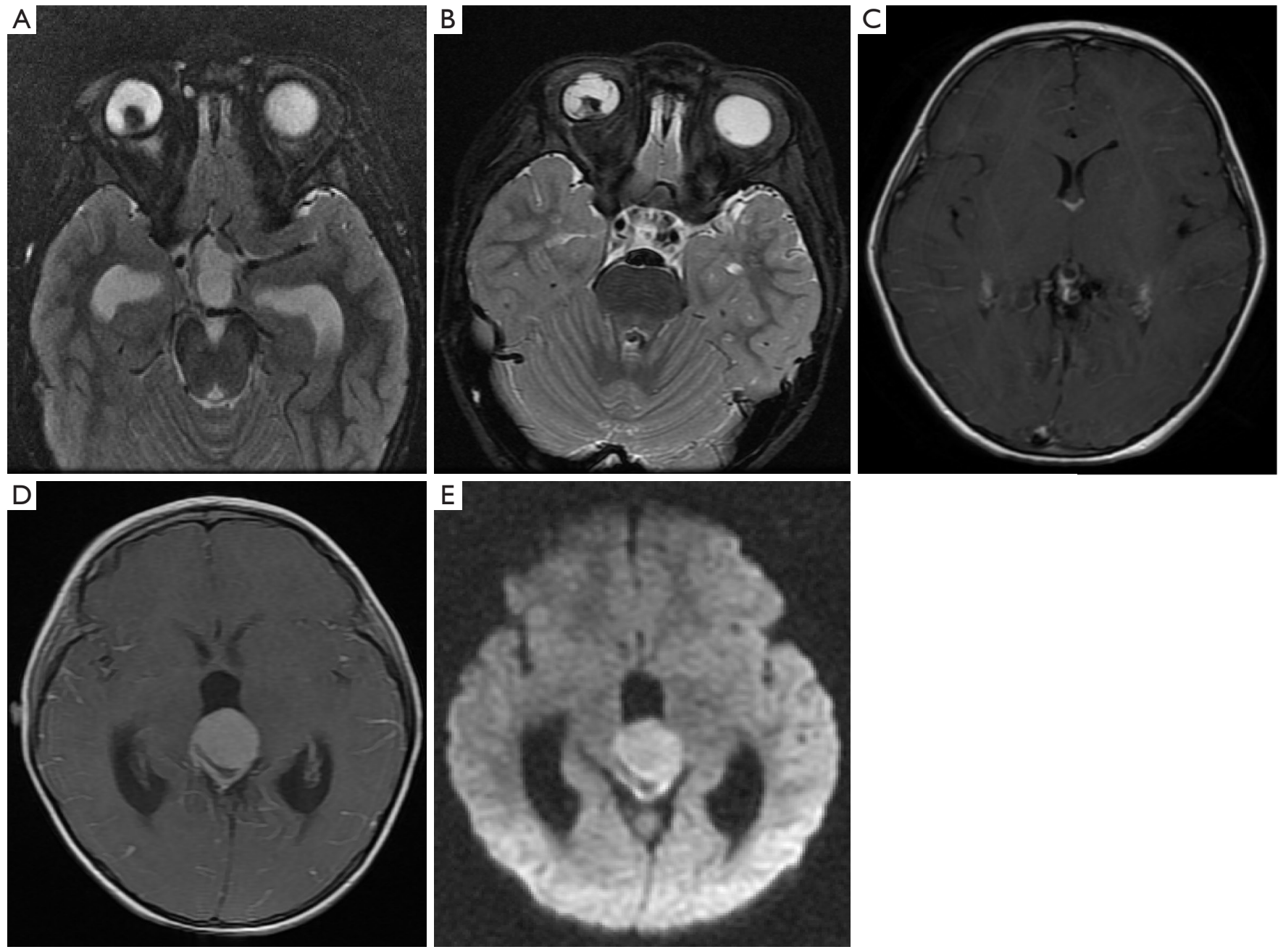

Figure 1 A girl was first diagnosed with bilateral RB (participant 8) at age 33 months. This participant had received 6 cycles of intravenous chemotherapy and 3 cycles of ophthalmic arterial chemotherapy. The bilateral intraocular lesions were subsequently stable (A and B). The pineal gland region showed a $4.5 \mathrm{~mm}$ ring reinforcement during the follow-up, which might have been a benign pineal cyst (C). After 26 months, MRI examination found a pineal tumor $(27 \mathrm{~mm})$ with obvious enhancement in post contrast-enhanced T1WI and diffusion restriction in DWI (D,E). RB, retinoblastoma; MRI, magnetic resonance imaging; DWI, diffusion-weighted imaging.

deaths due to chemotherapy.

\section{Discussion}

As the most common intraocular childhood malignancy, RB has a prevalence of 1 in 18,000 (1-4,9,18-20). It is caused by germinal and/or somatic mutations inactivating both alleles of $R B 1$ on chromosome $13 \mathrm{q} 14$ (16). TRB/QRB is rare, with an incidence of $3 \%$ of all cases and $8-10 \%$ of hereditary cases $(6-8,16)$. In our hospital, 12 participants with TRB, and 2 with QRB were found among 1,392 intraocular RB patients, and the incidence of TRB was $0.86 \%$ of all cases $(12 / 1,392)$. The incidence of QRB was even lower than that of TRB; in the past 8 years, the incidence of QRB was $0.14 \%$ $(2 / 1,392)$ in our hospital.

It has been reported in the literature that $95 \%$ of patients with TRB are aged $<5$ years. The average age at the initial diagnosis of intraocular tumors is $23-48$ months, while the age at diagnosis of intracranial lesions is comparatively later, with an interval of approximately 21 months $(14,21,22)$. The average age at first diagnosis of suprasellar TRB is earlier than that of pineal TRB by up to 12 months $(1,11,22)$. 

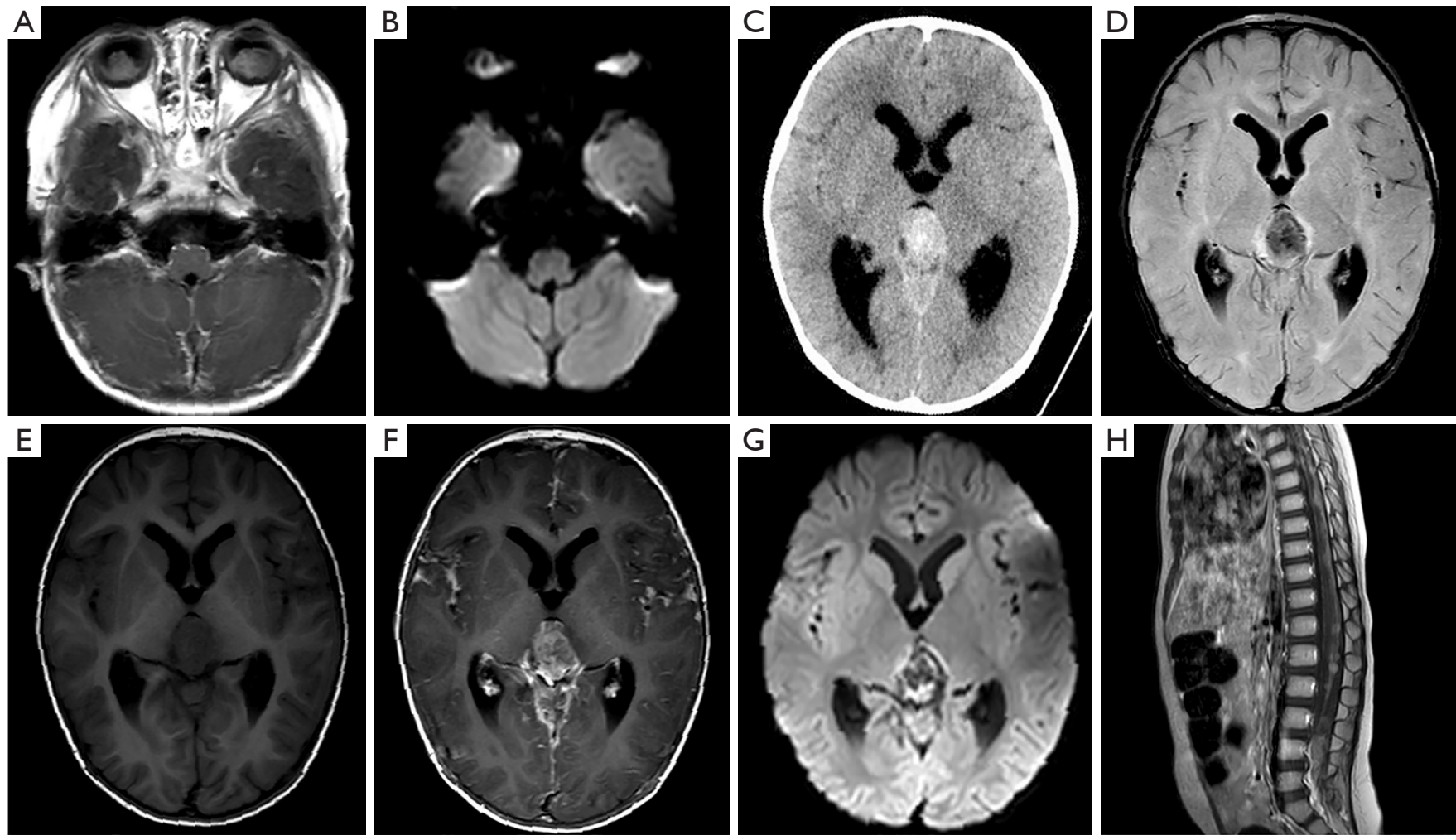

Figure 2 Pineal TRB with widespread cranial or spinal leptomeningeal disseminations in a 19-month-old girl (participant 1). Axial contrastenhanced T1WI (A) and DWI (B) showed bilateral RB in both eyes. The pineal mass showed high density in CT images (C), low signal intensity in T1WI (D) and T2WI (E), intense enhancement in post contrast-enhanced T1WI (F), and significant diffusion restriction in DWI (G). Axial and sagittal contrast-enhanced T1WI (A, F and H) showed widespread cranial or spinal leptomeningeal enhancement. TRB, trilateral retinoblastoma; CT, computed tomography; DWI, diffusion-weighted imaging.

In our study, all 14 participants were aged $<3$ years at first diagnosis, and intracranial lesions were also diagnosed at $<3$ years of age in 13 participants; only 1 patient was close to 5 years of age. Pineal and suprasellar TRB can occur simultaneously or can occur after the diagnosis of the intraocular tumor, and concurrent pineal or suprasellar TRB is always observed at a lower age, which may be because non-pineal TRB develops earlier than pineal disease and is, therefore, more likely to be diagnosed concurrently with RB $(1,12,17)$. In our study, the participants with concurrent TRB/QRB were $<30$ months old, whereas it was 37 months of age for later-onset TRB participants.

Children with hereditary RB usually have multifocal, bilateral $\mathrm{RB}$ and/or a family history or constitutional $R B 1$ mutation, whereas children with the somatic form generally have unilateral, unifocal disease, and negative family history (17). Approximately $89 \%$ of TRB patients have bilateral intraocular RB, and $11 \%$ have unilateral $\mathrm{RB}$
$(1,8,14,23,24)$. In our study, 13 participants $(92.9 \%)$ had bilateral RB, and 1 patient (7.1\%) had unilateral RB; all declared a negative family history. It should be noted that 1 participant had been misdiagnosed with unilateral RB associated with suprasellar and pineal tumors by MRI, and the "normal" eye by MRI was diagnosed with punctate RB classified as Group A by ophthalmoscopy. Previous reports have demonstrated that all multifocal, bilateral RBs are hereditary and progress to TRB. Some researchers have recently shown that up to $15 \%$ of sporadic unilateral $\mathrm{RB}$ cases may be hereditary, and these patients may also be at risk for the development of a second primary tumor at the intracranial midline $(8,25)$. In this study, a 2.5 -month-old girl was diagnosed with unilateral RB and a concomitant suprasellar tumor and showed extensive intracranial cerebrospinal fluid dissemination. Therefore, all unilateral/ bilateral RB patients must undergo routine MRI combined with ophthalmoscopy to prevent missed diagnoses of 

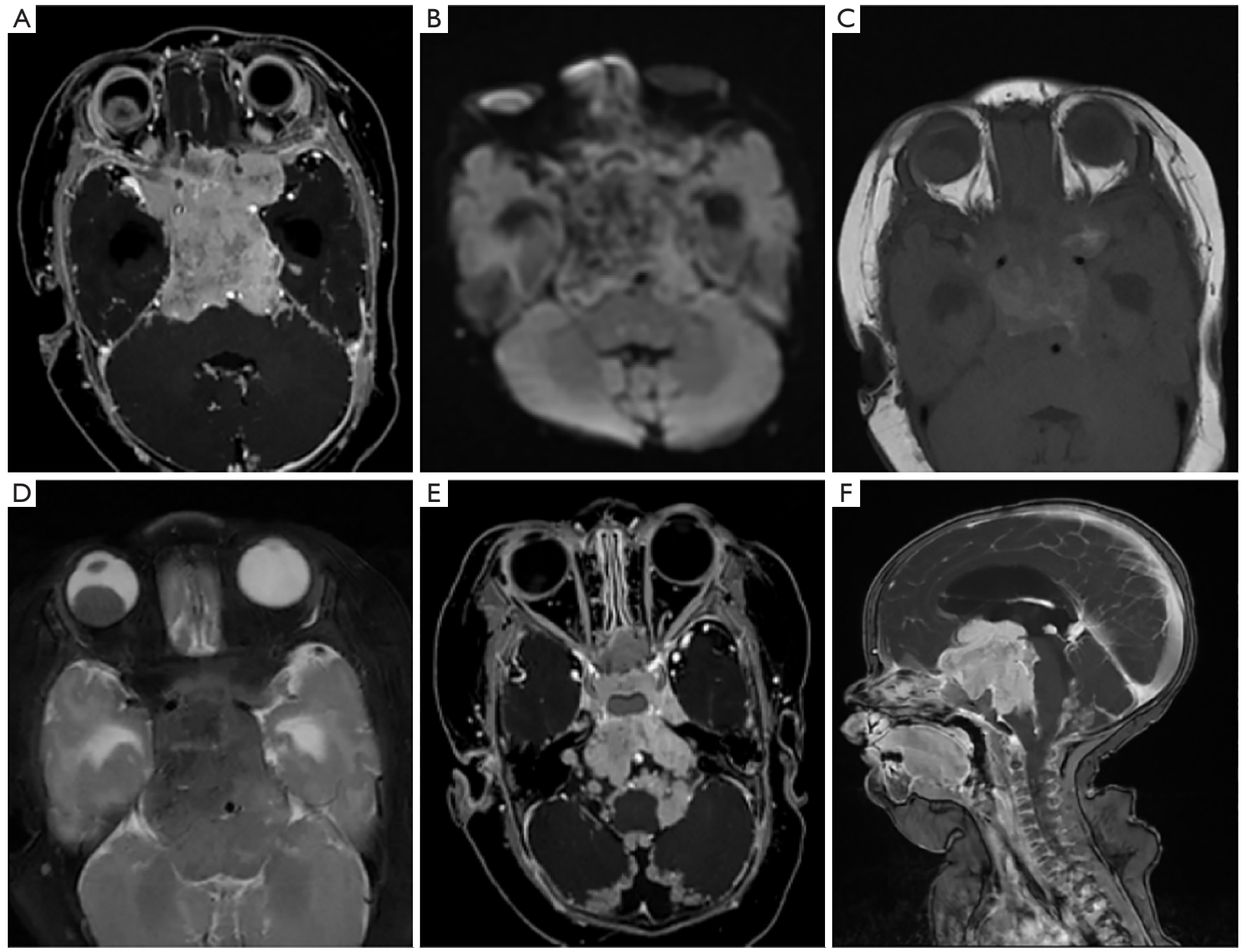

Figure 3 Suprasellar TRB with widespread cranial leptomeningeal dissemination in a 2.5-month-old girl (patient 5). Axial contrastenhanced T1WI demonstrated RBs in the right eye and the sellar region (A), and the sellar lesion showed significant restriction of diffusion in DWI (B). The suprasellar mass showed heterogeneous, slightly high signal intensity in T1WI (C) and low signal intensity in T2WI (D). Axial and sagittal contrast-enhanced T1WI (E and F) showed obvious enhancement in suprasellar tumors and widespread cranial leptomeningeal enhancement. TRB, trilateral retinoblastoma; RB, retinoblastoma; DWI, diffusion-weighted imaging.

intraocular or intracranial tumors. In our hospital, we recommend a long term and scheduled a follow-up for all $\mathrm{RB}$ patients, especially those with TRB, as follows: once every 3 months in the first 2 years, once every 6 months after 3-5 years, and once a year after 5 years. The follow-up duration should be $\geq 5$ years. During the follow-up period, the frequency should increase if the disease progresses. Concurrently, we optimized the orbital MRI scanning package for suspected or confirmed RB with a package including orbital T1WI, T2WI, DWI, orbital contrast- enhanced T1WI, and additional contrast-enhanced T1WI of the head, which is useful for the exclusion of pineal or suprasellar tumor and/or leptomeningeal dissemination.

The RB's intracranial lesion mostly occurs in the pineal region and $25 \%$ in the suprasellar area $(11,22)$. In our study, $50 \%(7 / 14)$ of intracranial tumors were located in the pineal region, $35.7 \%(5 / 14)$ in the suprasellar region, and $14.3 \%(2 / 14)$ in the pineal and suprasellar regions concurrently, which was classified as QRB. Similar to previously reported cases $(7-9,11,14-16,21,26)$, intracranial 

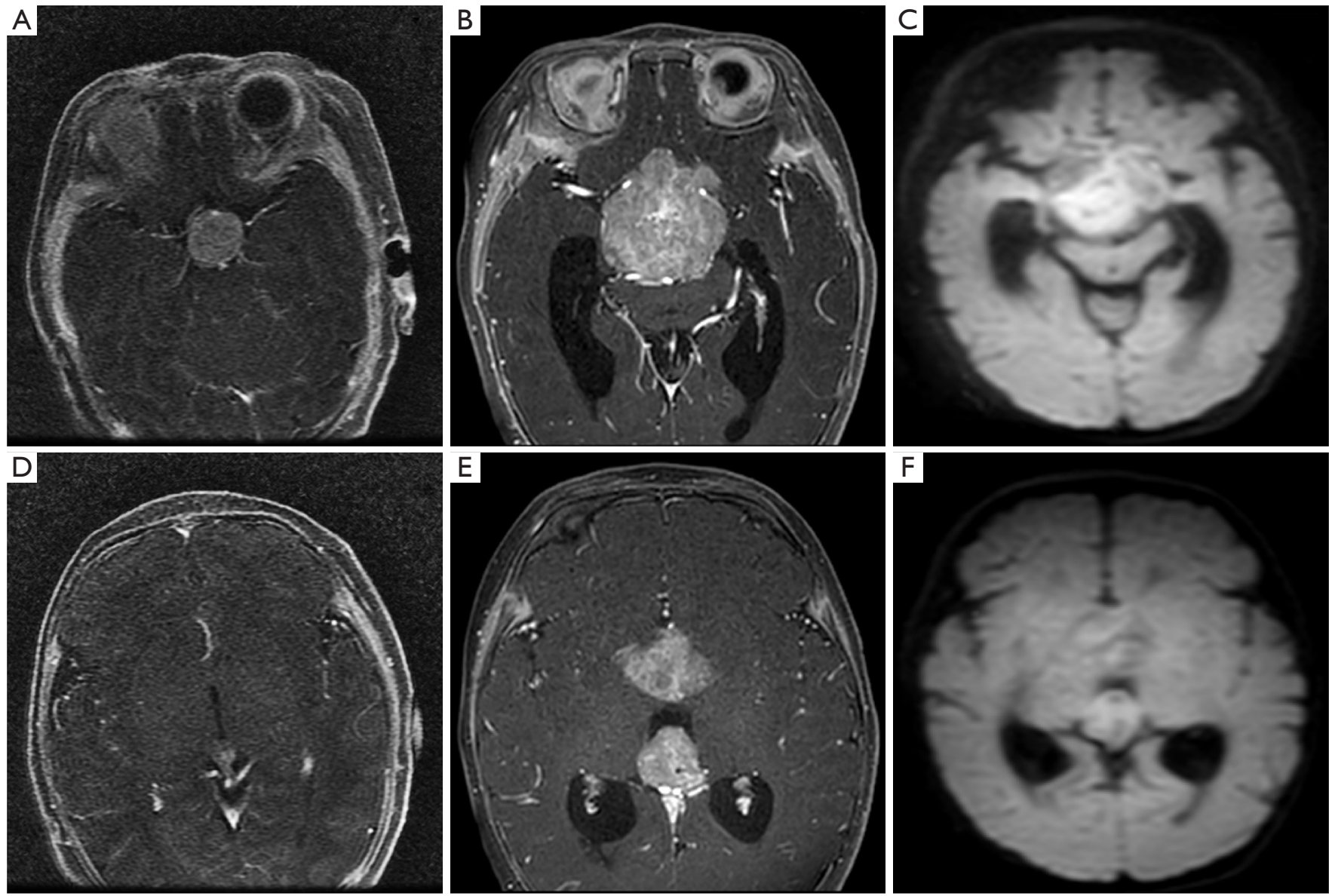

Figure $4 \mathrm{~A}$ boy was diagnosed with QRB with suprasellar and pineal lesions (the diameters of the lesions were $17 \mathrm{~mm}$ and $6 \mathrm{~mm}$; A and D) at 6 months of age (participant 6). However, the parents refused treatment. After 5 months, the suprasellar and pineal tumors showed obvious expansion (the diameters increased to $37 \mathrm{~mm}$ and $18 \mathrm{~mm}$, respectively) in axial contrast-enhanced T1WI and diffusion restriction in DWI (B, C, E and F). QRB, quadrilateral retinoblastoma; DWI, diffusion-weighted imaging.

lesions in our study showed a circumscribed round or oval mass in the pineal and/or suprasellar region. The lesions always demonstrated homogeneous medium signal intensity or heterogeneously mixed signal intensity in T1WI/ T2WI owing to intratumoral hemorrhage or calcification, intense enhancement in contrast-enhanced T1WI, and significant diffusion restriction in DWI with a low ADC of $(0.680 \pm 0.206) \times 10^{-3} \mathrm{~mm}^{2} / \mathrm{s}$, which was similar to the ADC value of pineal TRB reported by Farouk Sait et al. (27). The incidence of TRB is low, and the ADC value of intracranial lesions in our study can be used as a reference for diagnosis or tumor monitoring.

Although the 5 -year survival rate for patients with unilateral or bilateral $\mathrm{RB}$ is $>92 \%$, $\mathrm{RB}$ has a poor prognosis, with a 5 -year survival of $15.7 \%$, especially when the tumor spreads to the subarachnoid space $(9,15,17)$. Patients with concurrent TRB have higher 5-year survival than those whose disease is diagnosed $>3$ months after TRB $(47 \% v s$. $5.8 \%$, respectively) $(1,28,29)$. The difference may be due to the longer time interval for detecting the later-onset intracranial lesions, which allows the increase of tumor volume, cerebrospinal fluid spread, and death rate. Limited by follow-up time, we can only calculate up to the 3-year OS rate for our participants. The 1-, 2-, and 3-year OS rates were $80 \%, 18.75 \%$, and $12.5 \%$, respectively. Due to the small number of cases, it was impossible to calculate the 5-year survival rate of concurrent and later-onset TRB. Although TRB's 5-year survival rate is low, early detection of intracranial disease and increased use of high-dose chemotherapy, especially with stem cell rescue, can improve 

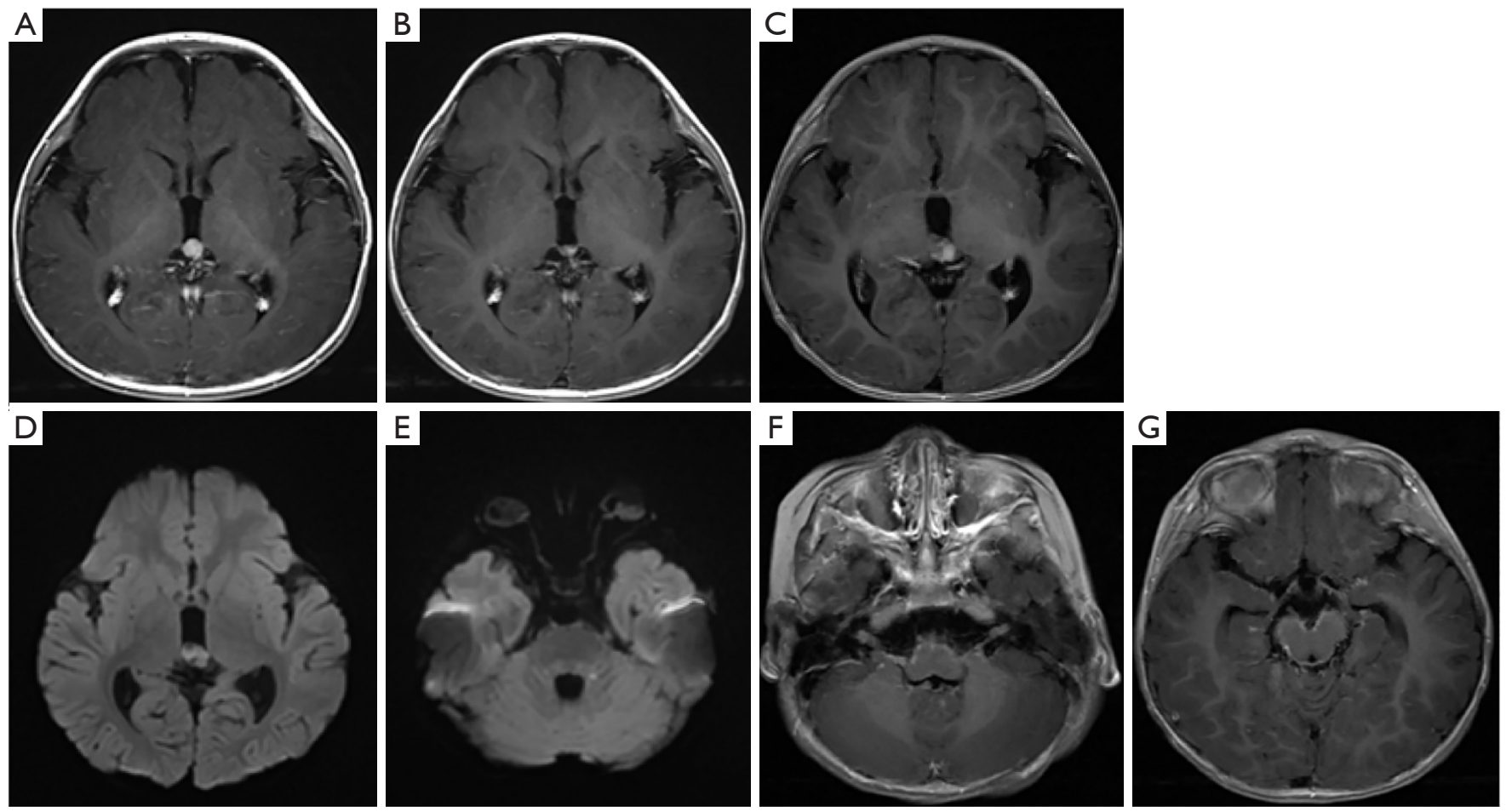

Figure 5 A 6.5-month-old boy was diagnosed with pineal (participant 11). The lesion in the pineal region had significantly shrunk after 9 cycles of intravenous chemotherapy and 2 cycles of ophthalmic arterial chemotherapy (A and B, the diameter of the lesion decreased from $8 \mathrm{~mm}$ to $3 \mathrm{~mm}$ ). The MRI follow-up after 8 months showed that the pineal tumor significantly enlarged (the diameter increased to $11 \mathrm{~mm}$ ) in axial contrast-enhanced T1WI with obvious restriction of diffusion in DWI (C and D). Concurrently, a high signal intensity nodule in DWI was found in the posterior inferior temporal wall of the left eye (E), and axial contrast-enhanced T1WI displayed leptomeningeal dissemination in the peri brainstem cistern and the bilateral internal auditory canals (F and G). TRB, trilateral retinoblastoma; MRI, magnetic resonance imaging; DWI, diffusion-weighted imaging.

survival $(1,17)$. In a previous study, all but one of the 22 long-term survivors received chemotherapy, and almost half underwent high-dose chemotherapy with stem cell rescue (1). In our study, 8 participants received chemotherapy. However, only the 2 participants who had simultaneously received high-dose chemotherapy and autologous hematopoietic stem cell rescue survived the whole follow-up period ( 24 and 54 months), which further demonstrated the great clinical benefit of autologous hematopoietic stem cell rescue.

Proton beam radiation therapy (PBRT) is a highly conformal type of radiation therapy and has gained increasing importance in oncology, especially for the treatment of pediatric tumors. The main benefit of protons over photons radiotherapy is the reduction of dose burden and, consequently, reducing the risk of long-term side effects. Several clinical studies have shown that PBRT for $\mathrm{RB}$ was effective even in patients suffering recurrence or showing resistance to chemotherapy, while few radiationassociated malignancies were noted (30-32). Therefore, PBRT seems to be an alternative disease-curing option for TRB patients, which may also reduce the risk of visual impairment and secondary malignancies in the foreseeable future.

In conclusion, TRB is rare and has a high mortality risk. Pineal or suprasellar TRB can occur simultaneously, or one can appear later. The intraocular and intracranial tumors showed similar MRI features with slightly different ADC values. Regular MRI scans and ophthalmoscopy for each RB patient should be used for early diagnosis and comprehensive assessment. High-dose chemotherapy with stem cell rescue can significantly improve patient survival. 

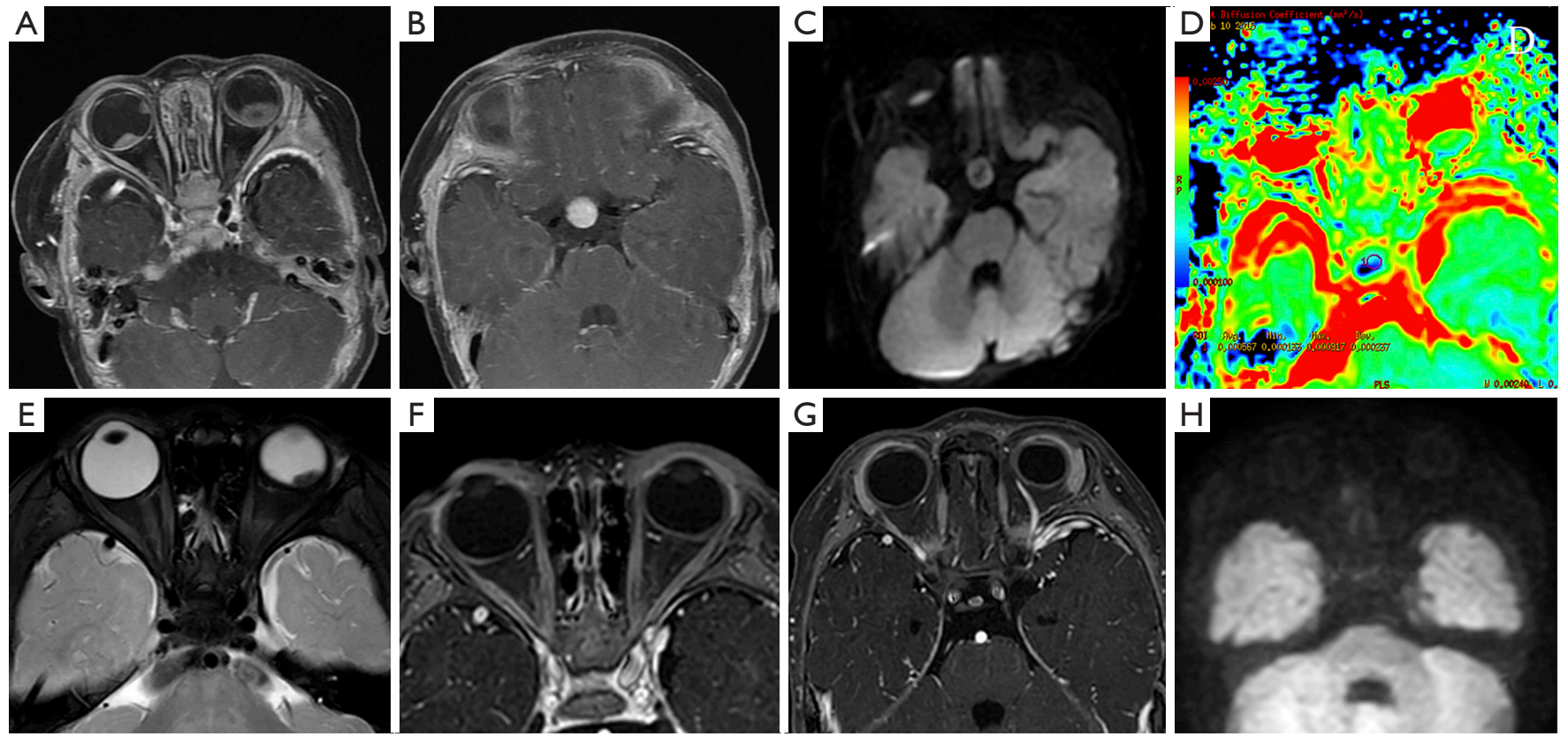

Figure 6 Suprasellar TRB in a 4-month-old boy (participant 14). Axial contrast-enhanced T1WI demonstrated multiple RBs in both eyes (A) and a suprasellar mass (B). The suprasellar mass showed intense enhancement in contrast-enhanced T1WI (B) and significant diffusion restriction in DWI (C) with an apparent diffusion coefficient of $0.620 \times 10^{-3} \mathrm{~mm}^{2} / \mathrm{s}(\mathrm{D})$. Both the intraocular (E and F) and suprasellar (G and $\mathrm{H})$ tumors had significantly shrunk after high-dose chemotherapy with stem cell rescue. The participant had survived with no recurrence by the last follow-up (54 months). TRB, trilateral retinoblastoma; RB, retinoblastoma; DWI, diffusion-weighted imaging.

\section{Acknowledgments}

Funding: This study was supported by the Science and Technology Commission of Shanghai Municipality (16411968300).

\section{Footnote}

Conflicts of Interest: All authors have completed the ICMJE uniform disclosure form (available at http://dx.doi. org/10.21037/qims-20-605). The authors have no conflicts of interest to declare.

Ethical Statement: The study was conducted in accordance with the Declaration of Helsinki and International Ethical Guidelines for Health-related Research Involving Humans. This study was approved by the Ethics Committee of Xinhua Hospital Affiliated to Shanghai Jiaotong University School of Medicine (XHEC-D-2020-124). Informed consent was provided by each participant or their responsible guardian.

Open Access Statement: This is an Open Access article distributed in accordance with the Creative Commons Attribution-NonCommercial-NoDerivs 4.0 International License (CC BY-NC-ND 4.0), which permits the noncommercial replication and distribution of the article with the strict proviso that no changes or edits are made and the original work is properly cited (including links to both the formal publication through the relevant DOI and the license). See: https://creativecommons.org/licenses/by-nc-nd/4.0/.

\section{References}

1. de Jong MC, Kors WA, de Graaf P, Castelijns JA, Kivela T, Moll AC. Trilateral retinoblastoma: a systematic review and meta-analysis. Lancet Oncol 2014;15:1157-67.

2. Dimaras H, Kimani K, Dimba EA, Gronsdahl P, White A, Chan HS, Gallie BL. Retinoblastoma. Lancet 2012;379:1436-46.

3. Chawla B, Sharma S, Sen S, Azad R, Bajaj MS, Kashyap S, Pushker N, Ghose S. Correlation between clinical features, magnetic resonance imaging, and histopathologic findings in retinoblastoma: a prospective study. Ophthalmology 2012;119:850-6. 
4. Rauschecker AM, Patel CV, Yeom KW, Eisenhut CA, Gawande RS, O'Brien JM, Ebrahimi KB, Daldrup-Link HE. High-resolution MR imaging of the orbit in patients with retinoblastoma. Radiographics 2012;32:1307-26.

5. Cui Y, Luo R, Wang R, Liu H, Zhang C, Zhang Z, Wang D. Correlation between conventional MR imaging combined with diffusion-weighted imaging and histopathologic findings in eyes primarily enucleated for advanced retinoblastoma: a retrospective study. Eur Radiol 2018;28:620-9.

6. Dunkel IJ, Jubran RF, Gururangan S, Chantada GL, Finlay JL, Goldman S, Khakoo Y, O'Brien JM, Orjuela M, Rodriguez-Galindo C, Souweidane MM, Abramson DH. Trilateral retinoblastoma: potentially curable with intensive chemotherapy. Pediatr Blood Cancer 2010;54:384-7.

7. Provenzale JM, Weber AL, Klintworth GK, McLendon RE. Radiologic-pathologic correlation. Bilateral retinoblastoma with coexistent pinealoblastoma (trilateral retinoblastoma). AJNR Am J Neuroradiol 1995;16:157-65.

8. Andrade GC, Pinto NP, Motono M, Chojniak MM, Chojniak R, Bezerra SM. Trilateral retinoblastoma with unilateral eye involvement. Rev Assoc Med Bras (1992) 2015;61:308-10.

9. Provenzale JM, Gururangan S, Klintworth G. Trilateral retinoblastoma: clinical and radiologic progression. AJR Am J Roentgenol 2004;183:505-11.

10. Richter S, Vandezande K, Chen N, Zhang K, Sutherland J, Anderson J, Han L, Panton R, Branco P, Gallie B. Sensitive and efficient detection of RB1 gene mutations enhances care for families with retinoblastoma. Am J Hum Genet 2003;72:253-69.

11. Paulino AC. Trilateral retinoblastoma: is the location of the intracranial tumor important? Cancer 1999;86:135-41.

12. Kivela T. Trilateral retinoblastoma: a meta-analysis of hereditary retinoblastoma associated with primary ectopic intracranial retinoblastoma. J Clin Oncol 1999;17:1829-37.

13. Amare P, Jose J, Chitalkar P, Kurkure P, Pai S, Nair C, Advani S. Trilateral retinoblastoma with an RB1 deletion inherited from a carrier mother: a case report. Cancer Genet Cytogenet 1999;111:28-31.

14. Marcus DM, Brooks SE, Leff G, McCormick R, Thompson T, Anfinson S, Lasudry J, Albert DM. Trilateral retinoblastoma: insights into histogenesis and management. Surv Ophthalmol 1998;43:59-70.

15. James SH, Halliday WC, Branson HM. Best cases from the AFIP: Trilateral retinoblastoma. Radiographics 2010;30:833-7.
16. D’Elia G, Grotta S, Del Bufalo F, De Ioris MA, Surace C, Sirleto P, Romanzo A, Cozza R, Locatelli F, Angioni A. Two novel cases of trilateral retinoblastoma: genetics and review of the literature. Cancer Genet 2013;206:398-401.

17. Yamanaka R, Hayano A, Takashima Y. Trilateral retinoblastoma: A systematic review of 211 cases. Neurosurg Rev 2019;42:39-48.

18. Corson TW, Gallie BL. One hit, two hits, three hits, more? Genomic changes in the development of retinoblastoma. Genes Chromosomes Cancer 2007;46:617-34.

19. Burkhart DL, Sage J. Cellular mechanisms of tumour suppression by the retinoblastoma gene. Nat Rev Cancer 2008;8:671-82.

20. Dimaras H, Khetan V, Halliday W, Orlic M, Prigoda NL, Piovesan B, Marrano P, Corson TW, Eagle RC Jr, Squire JA, Gallie BL. Loss of RB1 induces non-proliferative retinoma: increasing genomic instability correlates with progression to retinoblastoma. Hum Mol Genet 2008;17:1363-72.

21. de Jong MC, Kors WA, de Graaf P, Castelijns JA, Moll AC, Kivela T. The Incidence of Trilateral Retinoblastoma: A Systematic Review and Meta-Analysis. Am J Ophthalmol 2015;160:1116-26 e5.

22. Wright KD, Qaddoumi I, Patay Z, Gajjar A, Wilson MW, Rodriguez-Galindo C. Successful treatment of early detected trilateral retinoblastoma using standard infant brain tumor therapy. Pediatr Blood Cancer 2010;55:570-2.

23. de Jong MC, de Graaf P, Brisse HJ, Galluzzi P, Goricke SL, Moll AC, Munier FL, Popovic MB, Moulin AP, Binaghi S, Castelijns JA, Maeder P, European Retinoblastoma Imaging $\mathrm{C}$. The potential of $3 \mathrm{~T}$ high-resolution magnetic resonance imaging for diagnosis, staging, and follow-up of retinoblastoma. Surv Ophthalmol 2015;60:346-55.

24. de Jong MC, Moll AC, Goricke S, van der Valk P, Kors WA, Castelijns JA, de Graaf P. From a Suspicious Cystic Pineal Gland to Pineoblastoma in a Patient with Familial Unilateral Retinoblastoma. Ophthalmic Genet 2016;37:116-8.

25. Ibarra MS, O'Brien JM. Is screening for primitive neuroectodermal tumors in patients with unilateral retinoblastoma necessary? J AAPOS 2000;4:54-6.

26. Shah I, Baig A, Razzaq A, Faruqi A, Ali A, Khan FQ. Trilateral retinoblastoma with unilateral eye involvement. J Pak Med Assoc 2013;63:910-2.

27. Farouk Sait S, Haque S, Karimi S, Rebeiz KJ, Francis JH, Marr BP, Abramson DH, Souweidane MM, Dunkel IJ. A Potential Role For Apparent Diffusion Coefficient in the Diagnosis of Trilateral Retinoblastoma. J Pediatr Hematol 
Oncol 2020;42:238-43.

28. Kivela T. The epidemiological challenge of the most frequent eye cancer: retinoblastoma, an issue of birth and death. Br J Ophthalmol 2009;93:1129-31.

29. Kaliki S, Srinivasan V, Gupta A, Mishra DK, Naik MN. Clinical features predictive of high-risk retinoblastoma in 403 Asian Indian patients: a case-control study. Ophthalmology 2015;122:1165-72.

30. Jung EH, Kim JH, Kim JY, Jo DH, Yu YS. Outcomes of
Proton Beam Radiation Therapy for Retinoblastoma With Vitreous Seeds. J Pediatr Hematol Oncol 2018;40:569-73.

31. Thomas H, Timmermann B. Paediatric proton therapy. $\mathrm{Br}$ J Radiol 2020;93:20190601.

32. Chang JW, Yu YS, Kim JY, Shin DH, Choi J, Kim JH, Kim SJ. The clinical outcomes of proton beam radiation therapy for retinoblastomas that were resistant to chemotherapy and focal treatment. Korean J Ophthalmol 2011;25:387-93.
Cite this article as: Gui T, Zheng $\mathrm{H}$, Liu M, Xia Z, Ji X, Yin Q, Wang D, Li Y, Chen S. Clinical and magnetic resonance imaging features of 14 patients with trilateral retinoblastoma. Quant Imaging Med Surg 2021;11(4):1458-1469. doi: 10.21037/ qims-20-605 
Table S1 Patient Characteristics

\begin{tabular}{|c|c|c|c|c|c|c|c|c|c|c|c|c|}
\hline $\begin{array}{l}\text { Patient } \\
\text { number }\end{array}$ & Sex & Intraocular RB & $\begin{array}{l}\text { Age at Dx } \\
\text { (months) }\end{array}$ & $\begin{array}{l}\text { Months } \\
\text { from Dx to } \\
\text { trilateral }\end{array}$ & Trilateral site & $\begin{array}{l}\text { Intracranial tumor } \\
\text { size }(\mathrm{mm})\end{array}$ & $\begin{array}{l}\text { Intracranial } \\
\text { tumor ADC } \\
\#\left(\times 10^{-3} \mathrm{~mm}^{2} / \mathrm{s}\right)\end{array}$ & $\begin{array}{l}\text { Intraocular tumor } \\
\text { ADC }\left(\times 10^{-3} \mathrm{~mm}^{2} / \mathrm{s}\right) \\
(\mathrm{L} / \mathrm{R})\end{array}$ & $\begin{array}{l}\text { Metastases } \\
\text { seen at TRB } \\
\text { Dx }\end{array}$ & $\begin{array}{l}\text { Symptomatic } \\
\text { TRB }\end{array}$ & Chemotherapy & Status \\
\hline 1 & Female & Bilateral & 19 & 0 & Pineal & $22 \times 18 \times 14$ & 0.65 & $0.74 / 0.85$ & Yes & Yes & Not treated & Lost to follow-up \\
\hline 2 & Male & Bilateral & 4 & 0 & Pineal & $9.5 \times 5.5 \times 5$ & $N A^{*}$ & $N A^{*}$ & NO & NO & Not treated & DOD (18 months) \\
\hline 3 & Female & Bilateral & 16 & 0 & Suprasellar & $21 \times 15 \times 12$ & 1.25 & $0.84 / 0.82$ & NO & Yes & Not treated & Lost to follow-up \\
\hline 4 & Female & Bilateral & 11 & 0 & Suprasellar & $33 \times 26 \times 40$ & 0.57 & $0.66 / 0.67$ & Yes & Yes & Not treated & DOD (4 months) \\
\hline 5 & Female & Unilateral & 2.5 & 0 & Suprasellar & $40 \times 45 \times 43$ & 0.63 & No affected/ 0.41 & Yes & Yes & Not treated & DOD (3 months) \\
\hline 6 & Male & Bilateral & 6 & 0 & $\begin{array}{l}\text { Suprasellar + } \\
\text { pineal }\end{array}$ & $15 \times 14 \times 12 / 5 \times 4.2 \times 4$ & 0.77 & 0.29 / Atrophy & NO & NO & Not treated & DOD (8 months) \\
\hline 7 & Male & Bilateral & 26 & 0 & $\begin{array}{l}\text { Suprasellar + } \\
\text { pineal }\end{array}$ & $5 \times 4.5 \times 5 / 15 \times 13 \times 10$ & $N A^{*}$ & $N A^{*}$ & NO & NO & VPCyE & Lost to follow-up \\
\hline 8 & Female & Bilateral & 33 & 26 & Pineal & $27 \times 21 \times 22$ & 0.73 & $N A^{*}$ & NO & Yes & VPCyE & DOD (34 months) \\
\hline 9 & Male & Bilateral & 15 & 10 & Pineal & $15 \times 9 \times 7$ & $N A^{*}$ & $N A^{*}$ & NO & Yes & VPCyE & DOD (17 months) \\
\hline 10 & Male & Bilateral & 8 & 19 & Pineal & $12.5 \times 12 \times 13$ & 0.54 & $N A^{*}$ & NO & NO & VPCyE & DOD (13 months) \\
\hline 11 & Male & Bilateral & 6.5 & 0 & Pineal & $8 \times 6 \times 7$ & 0.52 & $0.55 / 0.73$ & NO & NO & VPCyE & DOD (18 months) \\
\hline 12 & Male & Bilateral & 12 & 0 & Suprasellar & $30 \times 22 \times 24$ & 0.53 & $0.58 / 0.74$ & NO & Yes & VPCyE & EFS (3 months) \\
\hline 13 & Female & Bilateral & 15 & 0 & Pineal & $11 \times 8 \times 9$ & 0.68 & $0.83 / 0.32$ & NO & NO & VPCyE, AHSCR & EFS (24 months) \\
\hline 14 & Male & Bilateral & 4 & 0 & Suprasellar & $10 \times 10 \times 7$ & 0.62 & $0.74 / 0.14$ & NO & NO & VPCyE, AHSCR & EFS (54 months) \\
\hline
\end{tabular}

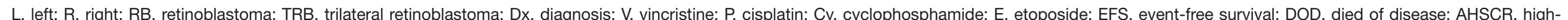

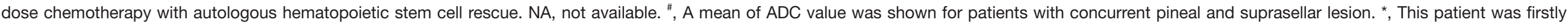
diagnosed in other hospital but treated and followed-up in Xinhua hospital. We failed to obtain the ADC value. 